\title{
Global Stability Analysis and Stabilization of Power Amplifier
}

\author{
Almudena Suárez \\ Communications Engineering Department \\ University of Cantabria \\ Santander, Spain \\ almudena.suarez@unican.es
}

\author{
Sanggeun Jeon, David Rutledge \\ California Institute of Technology \\ Pasadena, USA
}

\begin{abstract}
Power amplifiers often exhibit undesired behavior from certain input power value which cannot be predicted with a small-signal stability analysis. Among the commonly observed undesired phenomena are spurious oscillations, frequency divisions, hysteresis or chaos. In this contribution, simulation tools are presented, enabling an in-depth study of the origin and characteristics of these phenomena. The developed global stability analysis and stabilization tools have allowed an efficient suppression of the undesired behavior in a switching-mode power amplifier, with minimum degradation of the amplifier performance, in terms of drain efficiency and output power.
\end{abstract}

Auxiliary generator, parametric oscillation, pole-zero identification, power amplifiers, stability analysis.

\section{INTRODUCTION}

A power amplifier may be stable at small signal, fulfilling the stability criteria given by the Rollet factor and stability circles or other more complete ones, like those based on the use of the normalized determinant function [1]. However, as the input power increases, spurious oscillation, frequency divisions or a continuous spectrum of high power, associated to a chaotic solution, may be obtained. None of these phenomena can be predicted with a small-signal stability analysis of the power amplifier. This is because the instability arises from the largesignal regime obtained when increasing the input power [2]. It is generally due to nonlinear capacitances giving rise to an excess negative resistance together with resonance at a certain frequency value. In other cases, such as self-biased switchingpower amplifiers, the oscillation can be associated to the amplifier gain increase versus the input power, together with feedback effects. The described phenomena are difficult to predict when using harmonic balance $(\mathrm{HB})$. The problem comes from the fact that the periodic solution at the drive frequency is always a valid mathematical solution of the circuit, even when it is actually unstable and, thus, physically unobservable [3]. In HB, the circuit variables are represented in a Fourier series and a set of nonlinear algebraic equations at the different harmonic frequencies is solved through an errorminimization algorithm, very sensitive to the initial value. Due to the difficulty in accurately initializing the oscillation frequency and amplitude, convergence is obtained by default to the periodic solution with no oscillation at all. The problem can be overcome with a stability analysis of the obtained periodic solution. In order for the solution to be stable, any applied small perturbation must exponentially vanish in time. Whereas the stability analysis of a small-signal amplifier is carried out by linearizing the circuit about its DC solution, the stability analysis of a large-signal amplifier must be carried out by linearizing the circuit about the large-signal periodic regime resulting from the input drive [2,4-5]. This work will present an in-depth stability analysis of a Class $\mathrm{E} / \mathrm{F}_{\text {odd }}$ power amplifier, which exhibited anomalous behavior in the intermediate range of input power. The study will be carried out through the use of accurate stability and bifurcation analysis tools, applied in combination with HB. The aim is to provide full explanation of about the instability mechanisms leading to commonly observed undesired behavior in power amplifiers. As will be shown, this understanding is invaluable in order to devise an efficient global stabilization procedure of the amplifier circuit.

\section{ANOMALOUS EXPERIMENTAL BEHAVIOR OF THE CLASS- $\mathrm{E} / \mathrm{F}_{\text {ODD }}$ POWER AMPLIFIER}

The considered power amplifier contains two push-pull pairs with four vertical double-diffused MOSFETs (VDMOS) that are operated in Class-E/ $\mathrm{F}_{\text {odd }}$ mode at $29 \mathrm{MHz}$. (Fig. 1). The outputs of the four VDMOS transistors are combined using a distributed active transformer [6-7]. The capacitor $C_{\text {res }}$ and the transformer magnetization inductance build a parallel resonance at the operating frequency, and present appropriate tuning impedances to the transistors at the fundamental and odd harmonics for the Class-E/ $\mathrm{F}_{\text {odd }}$ operation.

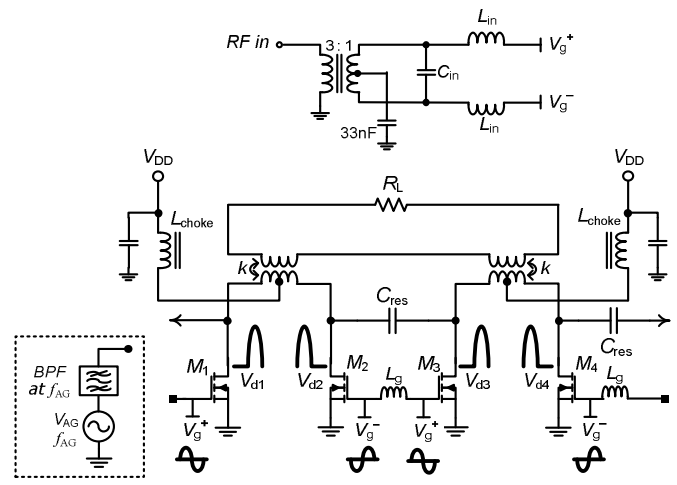

Figure 1. Schematic of the Class-E/ $\mathrm{F}_{\text {odd }} \mathrm{PA}$, with sketches of the voltage waveforms at the main circuit nodes.

Figure 2 presents the variation of the measured output spectrum versus the input power $P_{\text {in }}$ for the constant drain bias $V_{\mathrm{DD}}=72 \mathrm{~V}$. For low input power, only a leakage signal at the input frequency is obtained (Fig. 3a). The amplifier periodic solution is stable. However, at $P_{\text {in } 1}=5.5 \mathrm{~W}$, the output 
spectrum suddenly turns into the one in Fig. 3b. The continuity of this spectrum suggests chaotic (non-periodic) behavior. As the input power is further increased, this kind of spectrum continues to be observed until the input power reaches $P_{\text {in } \_2}=13.0 \mathrm{~W}$. From this value on, the spectrum becomes the proper one (Fig. 3c), with high drain efficiency and output power. Now the input power will be decreased from above $P_{\text {in } 2 \text {. At }} P_{\text {in } 2}$ the periodic spectrum becomes chaotic, with no hysteresis. However, the chaotic solution persists up to the power value $P_{\text {in } 3}=5.3 \mathrm{~W}$ at which a mixer-like spectrum is generated (Fig. 3d), never observed when increasing the power. It is a self-oscillating mixer regime, in which the input signal at $29 \mathrm{MHz}\left(f_{\text {in }}\right)$ mixes with a self-oscillation at about $4 \mathrm{MHz}\left(f_{\mathrm{a}}\right)$. If the input power is further reduced, the oscillation vanishes at the input power $P_{\text {in } 4}=5.0 \mathrm{~W}$ and the solution becomes periodic, with leakage output power.

\section{STABILITY ANALYSIS}

Initially, we will apply a stability analysis to the periodic solution at $f_{\text {in }}$ in order to determine the reasons why it is not experimentally observable for a certain $P_{\text {in }}$ interval. For the stability analysis, a small-amplitude perturbation must be considered [3]. This allows the linearization of the HB equations about the considered large-signal periodic solution. To obtain this linearization, a small-signal current generator is connected in parallel at a sensitive circuit node $n$, like those corresponding to the transistor terminals [3-4]. Because instabilities may occur at any frequency value, the frequency $f$ of this small-signal generator $I_{\mathrm{n}}$ is non-rationally related to $f_{\text {in }}$ (Fig. 6). The circuit operates in linear regime with respect to $I_{\mathrm{n}}(f)$, so, at each frequency $f$, its solution may be determined with the conversion-matrix approach [4-5]. The ratio $Z_{\text {in }}(f)=$ $V_{\mathrm{n}}(f) / I_{\mathrm{n}}(f)$ provides a single-input, single-output transfer function. All the closed-loop transfer functions that can be defined from a given linear system share the same denominator [5], given by the system characteristic determinant. Thus, any possible transfer function will contain the information about the system stability, in particular, $Z_{\text {in }}(f)$. To extract this stability information, the perturbation frequency $f$ is swept, applying pole-zero identification to $Z_{\text {in }}(f)$. Cancellations of unstable poles with zeroes of the same values may occur, so it is advisable to repeat the analysis for different locations of the current source [4-5]. The described stability analysis has been applied for the same bias conditions $V_{\mathrm{DD}}=72 \mathrm{~V}$ and $P_{\text {in }}$ range considered in the measurements, with $I_{\mathrm{n}}(f)$ introduced at the drain terminal of one of the four transistors. In a first stage, the steady-state periodic solution corresponding to the particular $P_{\text {in }}$ value is obtained with HB. In a second stage, the impedance function $Z_{\text {in }}(f)$ is determined with the conversion matrix approach. The frequency $f$ is swept and pole-zero identification is applied to the resulting frequency-dependent function. Fig. 4 shows the evolution versus the input power of the pair of complex-conjugate poles that are closer to the imaginary axis. The periodic solution is initially stable, but at $P_{\text {in } \_ \text {H } l}=6.1 \mathrm{~W}$, the pair of complex-conjugate poles crosses the imaginary axis to the right-hand side of the complex plane and the periodic solution becomes unstable. The crossing of the imaginary axis by a pair of complex conjugate poles at a frequency $f_{\mathrm{a}}$ nonharmonically related to $f_{\text {in }}$ corresponds to a Hopf bifurcation, associated to the generation of an oscillation at $f_{\mathrm{a}}$ which mixes with $f_{\text {in. }}$. As $P_{\text {in }}$ continues to increase, the poles move further to the right, turn, and cross the imaginary axis again, to the lefthand side, at the power value $P_{\text {in_Hu }}=13.5 \mathrm{~W}$ for which the oscillation vanishes.
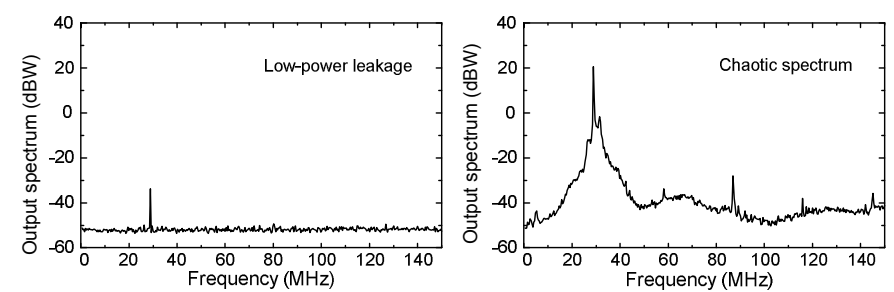

a)
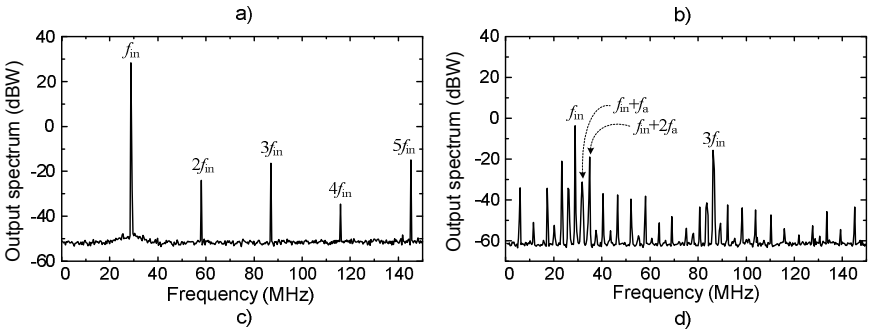

d)

Figure 2. Variation of the output spectrum. (a) $P_{\text {in }}=4 \mathrm{~W}$. (b) $P_{\text {in }}=10 \mathrm{~W}$. (c) $P_{\text {in }}=16.5 \mathrm{~W}$. (d) Power reduction. $P_{\text {in }}=5.2 \mathrm{~W}$.

Because the amplifier is expected to operate under different bias conditions, the next objective will be efficient determination of the set of $\left(V_{\mathrm{DD}}, P_{\text {in }}\right)$ values, with unstable behavior. This set of values will be delimited by the Hopfbifurcation locus, composed by the points at which the oscillation is generated or extinguished, depending on the sense of variation of the parameter. To obtain this locus, it is taken into account that the oscillation amplitude tends to zero at the Hopf-bifurcation points [3]. The oscillation condition, given by circuit total admittance equal to zero at any circuit node, should be fulfilled with oscillation amplitude tending to zero. Thus, at the Hopf bifurcation, the linear admittance $Y_{\text {in }}=1 / Z_{\text {in }}$ must be zero at the generated oscillation frequency $f=f_{\mathrm{a}}$. The Hopfbifurcation locus, which delimits the unstable behavior region in terms of $V_{\mathrm{DD}}$ and $P_{\mathrm{in}}$, is obtained by solving $Y_{\text {in }}\left(f_{\mathrm{a}}, V_{\mathrm{DD}}, P_{\text {in }}\right)=0$. Note that the oscillation frequency $f_{\mathrm{a}}$ must be included in the calculation, as this frequency is autonomous and thus varies along the locus. It is a twoequation system in three unknowns, giving a curve in the plane defined by $V_{\mathrm{DD}}$ and $P_{\text {in }}$. The application of the above technique to the Class-E/ $/ \mathrm{F}_{\text {odd }}$ amplifier provides the instability contour of Fig. 5. The consistency in the bifurcation points resulting from this analysis and the pole-zero identification should be noted. Experimental points have been superimposed.

In order to devise an efficient stabilization technique, the characteristics of the undesired self-oscillating mixer regime will be analyzed. The analysis will be carried out for $V_{\mathrm{DD}}$ and $P_{\text {in }}$ values inside the instability contour, where the oscillation amplitude is not small, with the aid of the auxiliary generator (AG) technique [3]. When choosing a voltage AG, this generator is connected in parallel at a circuit node. The AG operates at the oscillation frequency, i.e., $f_{\mathrm{AG}} \equiv f_{\mathrm{a}}$, and must be an open circuit at all other frequencies. Thus, an ideal bandpass filter is used in series with the AG (Fig. 1). The AG must not perturb the circuit steady-state solution, which is ensured by imposing $Y_{\mathrm{AG}}=I_{\mathrm{AG}} / V_{\mathrm{AG}}=0$, where $I_{\mathrm{AG}}$ and $V_{\mathrm{AG}}$ are the current 
and the voltage of the AG, respectively. For given $V_{\mathrm{DD}}$ and $P_{\text {in }}$, the amplitude $V_{\mathrm{AG}}$ and the frequency $f_{\mathrm{AG}}$ of the $\mathrm{AG}$ are calculated in order to fulfill the condition $Y_{\mathrm{AG}}\left(V_{\mathrm{AG}}, f_{\mathrm{AG}}\right)=0$. Even though the amplifier contains four transistors, only one AG, connected at one of the drain terminals, is necessary to determine the oscillating steady state. To investigate the nature of the oscillation, the phase at each drain terminal of the four transistors has been analyzed at different harmonic frequencies of the self-oscillating-mixer regime. At the oscillation frequency $f_{\mathrm{a}}$, the two transistors in the same push-pull pair are in phase, whereas the two pairs are $180^{\circ}$ out of phase. However, at the input-drive frequency $f_{\text {in }}$, the original phase-shift relationships are maintained, i.e., $180^{\circ}$ phase shift between the two transistors in the same pair and $180^{\circ}$ phase shift between the two pairs as well. Because the two transistors of a same push-pull pair are in phase at the oscillation frequency, the middle point of the pair becomes a virtual open at this frequency. This knowledge will be very helpful, in order to devise a suitable stabilization technique, involving a minimum degradation of the original circuit performance.

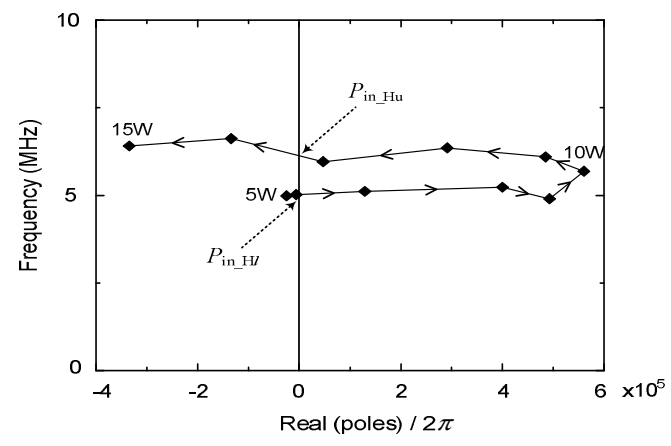

Figure 3. Pole locus versus the $P_{\text {in }}$ for $V_{D D}=72 \mathrm{~V}$.

The self-oscillating mixer solution at the two fundamental frequencies $f_{\text {in }}$ and $f_{\mathrm{a}}$ has been analyzed versus the input power $P_{\text {in }}$, for constant $V_{\mathrm{DD}}=72 \mathrm{~V}$, solving the equation $Y_{\mathrm{AG}}=0$ at each step. The resulting variation of the oscillation amplitude at the drain terminal, agreeing with $V_{\mathrm{AG}}$, is represented in Fig. 5. The curve exhibits a turning point $T$, responsible for the experimentally observed hysteresis phenomenon. Actually, when increasing $P_{\text {in }}$, the transition from stable amplifier behavior to the self-oscillating mixer regime ( $J_{1}$ in Fig. 5$)$ is due to the Hopf bifurcation $H^{1}{ }_{l}$ occurring in the amplifier solution. When $P_{\text {in }}$ is decreased, the jump back to the stable amplifier behavior $\left(J_{2}\right)$ is due to the turning point $T$ in the selfoscillating mixer solution. Note that the simulated hysteresis interval, in terms of the input power, is in good correspondence with the experimental one, observed in Fig. 2. The hysteresis phenomenon is well predicted by Fig. 5 . However, in the measurement, an abrupt transition from stable amplifier behavior to the chaotic regime occurred when increasing the input power (see Fig.2b). For superior analysis flexibility, the envelope transient [8] has been used. The circuit variables are expressed in a Fourier series with time-varying coefficients and a differential-equation system is solved in terms of these coefficients. Its application to autonomous circuits requires a proper initialization of the oscillation, which is carried out through the connection of an AG to the circuit at the initial time $\mathrm{t}_{0}$ only [7]. From the point $H_{1}^{2}$ in Fig. 5, the magnitude of the harmonic components $X_{\mathrm{k}, 1}(\mathrm{t})$ becomes time-varying (Fig. 4). The envelopes oscillate at a few hundred $\mathrm{kHz}$. Thus, there is a second oscillation, in addition to the previous oscillation at about $4 \mathrm{MHz}\left(f_{\mathrm{a}}\right)$. The Hopf bifurcation $\mathrm{H}^{2}$ gives rise to a second oscillation frequency $f_{\mathrm{a}}$, incommensurate with both $f_{\text {in }}$ and $f_{\mathrm{a}}$, so a three-fundamental regime is obtained at $H^{2}{ }_{l}$. Threefundamental solutions are likely to break into chaos [9], which explains the chaotic spectrum observed in the experiment (Fig. 2b). The interval in which the self-oscillating mixer solution at $f_{\text {in }}$ and $f_{\mathrm{a}}$ is unstable has been indicated with stars in Fig. 5. This bifurcation diagram is in good correspondence with all the different phenomena observed in the measurements.

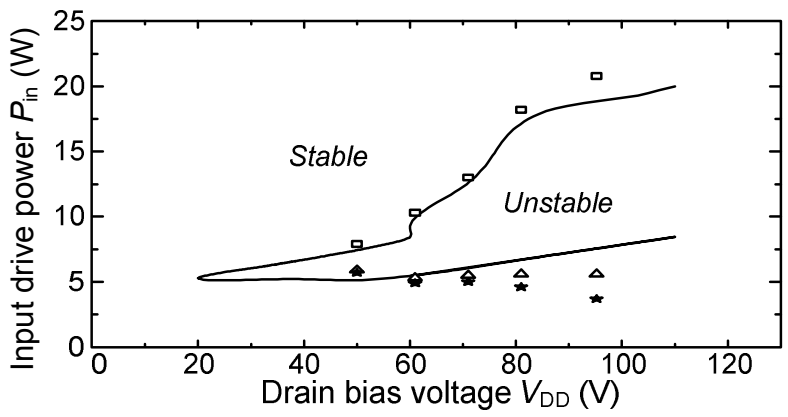

Figure 4. Instability contour in the plane defined by the drain bias voltage $\mathrm{V}_{\mathrm{DD}}$ and the input power $\mathrm{P}_{\text {in }}$.

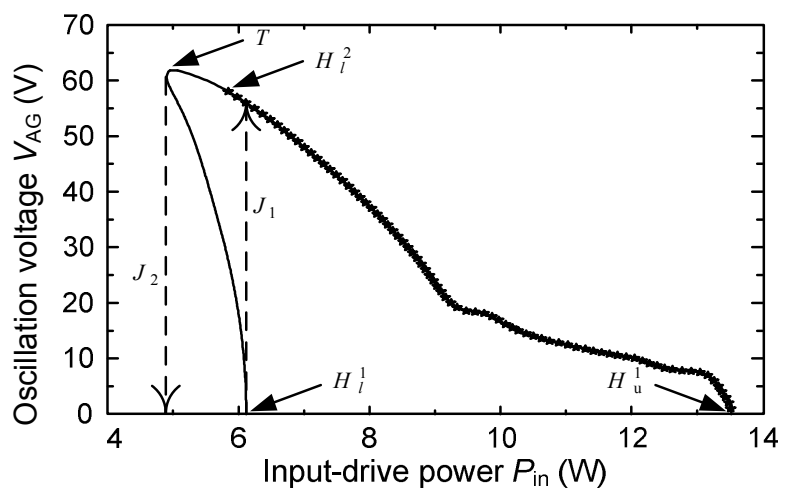

Figure 5. Quasi-periodic solution at $\mathrm{f}_{\mathrm{in}}$ and $\mathrm{f}_{\mathrm{a}}$. Variation of the oscillation amplitude versus $P_{\text {in }}$.

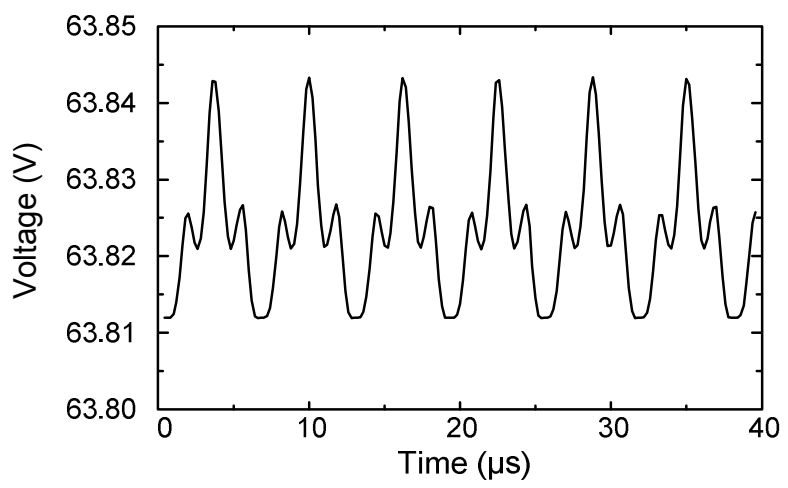

Figure 6. Time-varying envelopes obtained in the input-power interval indicated with stars in Fig. 5. 


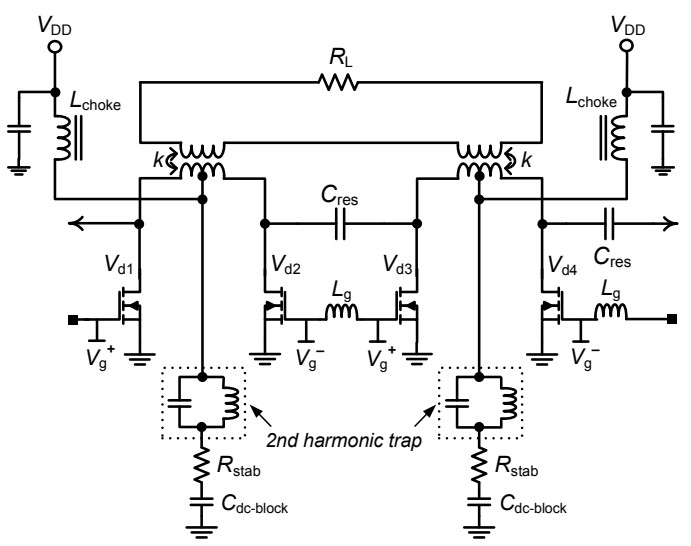

Figure 7. Circuit containing the stabilization network.

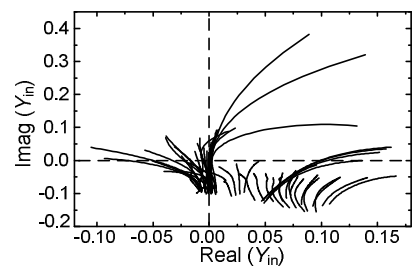

a)

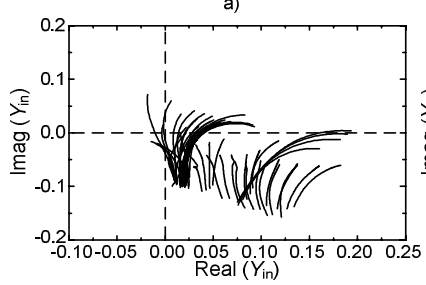

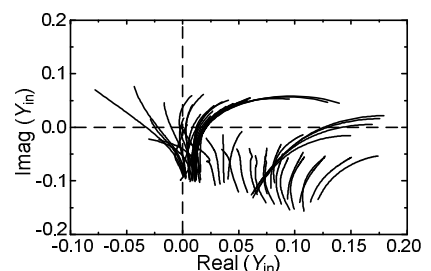

b)

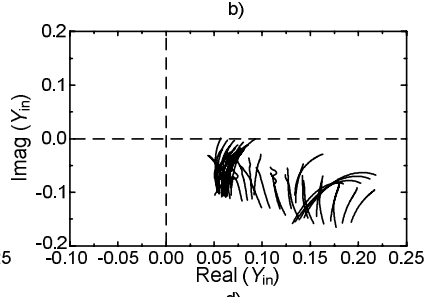

Figure 8. Determination of of the stabilization resistance, obtained through a double sweep in the two circuit parameters $V_{D D}$ and $P_{\text {in }}$. (a) Original circuit. (b) $\mathrm{R}=100 \mathrm{Ohm}$. (c) $\mathrm{R}=50 \mathrm{Ohm}$. (d) $\mathrm{R}=15 \mathrm{Ohm}$. Global stability of the power amplifier.

\section{Circuit Stabilization}

After understanding the different instability phenomena, the goal will be the global stabilization of the power amplifier for all the expected operation conditions, in terms of $V_{\mathrm{DD}}$ and $P_{\text {in }}$. From the phase analysis of the different frequency components of the undesired self-oscillating solution, the middle node of the push-pull pair behaves as a virtual open at the oscillation frequency. Thus, a simple means to stabilize the amplifier will be the addition of a resistor at this node to increase the positive resistance of the equivalent parallel-resonance circuit. This resistance will have little effect at the input-drive frequency because this middle node is a virtual short circuit at this frequency. The node should be an ideal open circuit at all the even harmonics of $f_{\text {in }}$, so a second harmonic trap is also connected in series (Fig. 7). The next step will be the determination of the stabilization-resistance value to ensure stable operation for the entire $V_{\mathrm{DD}}$ and $P_{\text {in }}$ ranges. Comparing the frequency variation of $Y_{\text {in }}$ with the pole-zero identification, it has been possible to associate the instability with the existence of negative conductance at the oscillation frequency, together with a zero crossing of the susceptance with positive slope. Different resistance values have been tested and, for

each, a double sweep has been performed in $\left(V_{\mathrm{DD}}, P_{\text {in }}\right)$. For each $\left(V_{\mathrm{DD}}, P_{\text {in }}\right)$, a HB calculation is carried out, together with a sweep in the current-source frequency $f$ using conversion matrix, between $3 \mathrm{MHz}$ and $5.5 \mathrm{MHz}$. This yields the input admittance $Y_{\text {in }}(f)$ seen by the current source. For each resistance value, the double sweep in $\left(V_{\mathrm{DD}}, P_{\text {in }}\right)$ provides a family of polar plots. For global stability, no crossing of the negative real semi-axis, with positive increase of the susceptance must be obtained [10]. Fig. 8 illustrates the application of this technique for three resistance values: $100 \Omega, 50 \Omega$, and $15 \Omega$. As expected, larger stability ranges are achieved as the stabilization resistance is reduced. For $R_{\text {stab }}=15 \Omega$, the amplifier becomes stable for all the expected operation values of $V_{\mathrm{DD}}$ and $P_{\text {in. }}$. We never observed in the measurement any oscillation or chaotic regime. The only significant degradation of the drain efficiency was $1.3 \%$ for $240 \mathrm{~W}$ output power.

\section{CONCLUSIONS}

This contribution shows how recently-developed stabilityanalysis tools can be used for a helpful understanding and suppresion of instability phenomena in power amplifiers. The techniques, based on harmonic balance, have been illustrated by means of their application to Class-E/F amplifier, at 29 $\mathrm{MHz}$, which exhibited self-oscillation, hysteresis and chaos. An in-depth analysis of the oscillation mechanism has also been carried out. From this study, a simplified equivalent circuit at the oscillation frequency has been derived, enabling an efficient determination of the topology and location of the required stabilization network. The circuit has been globally stabilized for all the expected values of drain-bias voltage and input power.

\section{REFERENCES}

[1] A. Platzker, W. Struble, "A rigorous yet simple method for determining stability of linear N-ports networks" GaAs IC Symposium Digest, pp. 251-254, 1993

[2] S. Mons, J.-C. Nallatamby, R. Queré, P. Savary, and J. Obregon, “A unified approach for the linear and nonlinear stability analysis of microwave circuits using commercially available tools," IEEE Trans. Microwave Theory \& Tech., vol. 47, no. 12, pp. 2403-2409, Dec. 1999.

[3] A. Suárez and R. Queré, Global Stability Analysis of Microwave Circuits, Artech House, Boston, 2003.

[4] J. Jugo, J. Portilla, A. Anakabe, A. Suárez, and J. M. Collantes, "Closedloop stability analysis of microwave amplifiers," IEE Electronics Letters, vol. 37, pp. 226-228, Feb. 2003.

[5] J. Jugo, A. Anakabe, and J. M. Collantes, "Control design in the harmonic domain for microwave and RF circuits," IEE Proc. Control Theory and Applications, vol. 150, no. 2, pp. 127-131, Mar. 2003.

[6] D. Kee, I. Aoki, A. Hajimiri, and D. B. Rutledge, "The class-E/F family of ZVS switching amplifiers," IEEE Trans. Microwave Theory \& Tech., vol. 51, no. 6, pp. 1677-1690, Jun. 2003.

[7] S. Jeon, A. Suárez, R. Rutledge, "Global stability analysis and stabilization of a Class-E/F amplifier with a distributed active transformer", IEEE Tran. Microwave Theory \& Tech, vol. 53, no. 12, Dec. 2005, pp. 3712- 3722 .

[8] E. Ngoya and R. Larcheveque, "Envelope transient analysis: a new method for the transient and steady state analysis of microwave communication circuits and systems," IEEE MTT-S, S. Francisco, Jun. 1996, pp. 1365-1368.

[9] J. M. T. Thompson and H. B. Stewart, Nonlinear Dynamics and Chaos, John Wiley \& Sons, 1986.

[10] K. Kurokawa, "Some basic characteristics of broadband negative resistance oscillator circuits," Bell Syst. Tech. J., vol. 48, pp. 1937-1955, Jul.-Aug. 1969. 